\title{
EI Trabajo Matemático de Profesores en el Tránsito de la Geometría Sintética a la Analítica en el Liceo
}

\section{The Mathematical Work of Teachers in the Transition from the Synthetic Geometry to the Analytic at Secondary School}

\author{
Carolina Henríquez-Rivas* \\ Elizabeth Montoya-Delgadillo**
}

\begin{abstract}
Resumen
En esta investigación se muestran resultados de un estudio con profesores de matemática de liceo en Chile, en temáticas de geometría euclidiana que articulan los enfoques sintético y analítico. El estudio presenta una situación de referencia que favorece ciertas rutas de trabajo específicas. La investigación se sustenta en el enfoque Espacio de Trabajo Matemático y estudia el trabajo personal del profesor con un enfoque metodológico cualitativo. La evidencia empírica proporciona elementos que permiten caracterizar los paradigmas en el subdominio cartesiano, lo cual ha sido un aporte al constructo teórico.
\end{abstract}

Palabras clave: Espacio de Trabajo Geométrico. Enfoque Sintético. Enfoque Analítico.

\begin{abstract}
The results of a research about Chilean teachers of Mathematics are shown in this investigation, in subject matters of Euclidean geometry, which articulates the approaches synthetically and analytically. The study presents a situation of reference that favors certain specific phases of work. The investigation sustains in the approach Mathematical Working Space and studies the personal work of the teacher with a methodological qualitative approach. The empirical evidence provides elements that allows us to characterize the paradigms in the cartesian sub-domain, which has been a contribution to the theoretical frame.
\end{abstract}

Keywords: Geometric working space, Synthetic Approach, Analytical Approach.

\section{Introducción}

En la presente investigación se hace referencia al trabajo geométricode profesores de liceo en Chile, relativo a temáticas insertas en el dominio de la geometría (euclidiana),

\footnotetext{
* Dra. Didáctica de la Matemática Pontificia Universidad Católica de Valparaíso (PUCV). Postdoctorado Instituto de Matemáticas, PUCV, Valparaíso, Chile. Dirección para correspondencia: Avenida Brasil 2950, Valparaíso, Chile. E-mail: carolina.henriquez@pucv.cl

${ }^{* * *}$ Dra. Didactique des Mathématiques Université Paris Diderot. Profesora Instituto de Matemáticas, PUCV, Valparaíso, Chile. Dirección para correspondencia: Avenida Brasil 2950, Valparaíso, Chile. E-mail: elizabeth.montoya@pucv.cl
} 
abarcando los enfoques con y sin coordenadas cartesianas -usamos la denominación geometría analítica y geometría sintética, respectivamente-. Específicamente, se presentan resultados relativos al diseño y experimentación de una situación de referencia en el espacio de trabajo geométrico personal de un profesor de secundaria. El trabajo se sustenta en la teoría Espacio de Trabajo Matemático, ETM,(KUZNIAK, 2011) en el dominio de la geometría.

Para diferenciar entre geometría analítica y geometría sintética se considera la distinción propuesta por Klein (1908, p. 73), quien señala “Geometría sintética, aquella en la cual se estudian las figuras en sí mismas sin intervención alguna de fórmulas, mientras que en la analítica, estas se aplican constantemente mediante el uso de los sistemas de coordenadas". A lo anterior, Klein (1908, p. 74) prosigue:

\begin{abstract}
En realidad, la diferencia entre ambas especies de Geometría es puramente cuantitativa: según predominen las fórmulas o las figuras, se tiene una $u$ otra Geometría, ya que una Geometría analítica no puede, sin perder su nombre, prescindir en absoluto de la representación geométrica, ni, por el contrario, la Geometría sintética puede ir más lejos sin expresar de un modo preciso, con fórmulas adecuadas sus resultados.
\end{abstract}

Además, en el célebre Programa de Erlangen (1872), Klein preconiza el cese a las disputas entre las geometrías sintética y analítica, pues bajo este punto de vista, una geometría vendría a ser el conjunto de propiedades invariantes mediante las transformaciones del grupo correspondiente (BOURBAKI, 1969).

En un estudio sobre la enseñanza de la geometría desarrollado por Gascón (2002), el autor da cuenta, entre otros aspectos, la falta de tránsito y complementariedad entre la geometría sintética y la analítica; para Gascón estas geometrías viven en mundos separados.

El presente trabajo se suscribe al punto de vista de Gascón, sustentando los análisis con evidencia empírica y una propuesta didáctica que muestra la necesidad que ambos enfoques geométricos se influencien mutuamente. Para lograr dicho propósito se proponen situaciones de referencia que buscan favorecer un $\mathrm{ETM}_{\mathrm{G}}$ personal eficiente en términos del tránsito y complementariedad de un enfoque a otro. En este artículo se presentan los resultados relativos al diseño, experimentación y análisis posteriores de un caso, correspondiente a un futuro profesor en último año de formación inicial.

\title{
2 Marco teórico
}

Este estudio se sustenta en el constructo del Espacio de Trabajo Matemático (KUZNIAK, 2011) en el dominio de la geometría $\left(\mathrm{ETM}_{\mathrm{G}}\right)$, el cual fue conocido inicialmente 
como Paradigmas Geométricos y Espacio de Trabajo Geométrico desarrollado por de Houdement y Kuzniak (1999, 2006).

\subsection{Espacio de trabajo matemático}

El ETM se define como un ambiente organizado que permite el trabajo de las personas que resuelven tareas matemáticas, el cual se constituye por dos planos, cognitivo y epistemológico, en relación directa con los objetos matemáticos del dominio en juego. En cada plano hay tres componentes: en el plano cognitivo están presentes los procesos de visualización, construcción y prueba; y en el epistemológico, el representamen, artefactos y referencial. Las componentes de los planos se articulan mediante tres génesis: semiótica, instrumental y discursiva. La génesis semiótica, asociada a las representaciones de los objetos matemáticos; la génesis instrumental, permite hacer operatorios los artefactos en el proceso constructivo; y la génesis discursiva da sentido al referencial teórico (definiciones, propiedades) para ponerlo al servicio del razonamiento matemático (KUZNIAK, 2011).

La idea del representamen está relacionada a la noción signo de Peirce (1978), y tiene que ver con el objeto matemático bajo formas más o menos abstractas: íconos, índices y símbolos. Un signo remite a su objeto de alguna de estas tres formas, según un proceso semiótico -llamado aquí visualización - involucrado en función de las significaciones de su utilizador. La articulación entre ambas componentes se produce con la génesis semiótica.

La componente artefactos se relaciona con la concepción de Rabardel (1995), quien distingue que un artefacto puede ser material o un sistema simbólico empleado como un medio para la acción. El instrumento es considerado como una entidad mediadora, entre el sujeto y el objeto sobre el cual se dirige la acción, y como una entidad mixta que comprende el artefacto y componentes cognitivos relacionados con los esquemas de uso. En el plano cognitivo el proceso de construcción está determinado por los instrumentos utilizados, y la articulación entre ambas componentes se produce con la génesis instrumental (ARTIGUE, 2002; TROUCHE, 2002).

La componente referencial remite a los elementos de índole teórico matemático del dominio en juego (geometría, álgebra, análisis, etc.). Aquí se encuentran las propiedades y definiciones involucradas en un razonamiento y cómo estas son utilizadas en un discurso para argumentar y convencer según un proceso de prueba. La articulación entre ambas componentes se produce con la génesis discursiva. 
En los distintos ETM es posible identificar tres tipos dependiendo de la función de la reflexión del sujeto cuando se enfrenta a un problema determinado: un referencial definido de manera ideal sobre criterios matemáticos (ETM de referencia), un espacio definido en términos de enseñanza en una institución dada con una función definida (ETM idóneo), y un espacio propio de cada utilizador conforme la reflexión de sus conocimientos matemáticos utilizados para resolver una tarea (ETM personal). En esta investigación los análisis están referidos principalmente al ETM personal del profesor.

\subsection{Paradigmas geométricos y espacio de trabajo geométrico}

Al considerar las particularidades del dominio geométrico en el ETM, la génesis semiótica se denomina génesis figural, la cual permite describir el proceso semiótico que está asociado al pensamiento visual que opera en geometría (KUZNIAK, 2011). El espacio real y local se presenta como componente particular del $\mathrm{ETM}_{\mathrm{G}}$ en el plano epistemológico (el representamen del ETM). El aspecto real es el acceso a los objetos como el resultado de la abstracción del modelo, y el aspecto local concierne al trabajo con una parte del modelo.

Para analizar las componentes de los planos del $\mathrm{ETM}_{\mathrm{G}}$ se han incluido enfoques que lo complementan. En relación a la visualización, para Duval (1995, 1999) este proceso está basado en la producción de una representación semiótica (figuras geométricas, gráficos cartesianos) de un objeto. Duval (2005) distingue dos modos de visualizar que pueden funcionar según el tipo de operación con las figuras y cómo se movilizan sus propiedades; un modo icónico y uno no-icónico.

Relativo a la componente artefactos se considera la concepción (ya citada) de Rabardel (1995), los cuales pueden ser de origen material, como una regla, un compás o un software, y que permiten trabajar sobre una figura. O bien, estos pueden ser simbólicos, y permiten producir un resultado en un registro semiótico distinto al figural. El enfoque instrumental (ARTIGUE, 2002; TROUCHE, 2002) favorece el estudio de una tarea específica dada a los alumnos en contextos tecnológicos, y ayuda a comprender el desarrollo del trabajo geométrico y sus dificultades asociadas al uso de las tecnologías. En este contexto, el trabajo de Gómez-Chacón y Kuzniak (2011) ofrece un estudio al trabajo geométrico de futuros profesores cuando utilizan un software geométrico (Geogebra) y cómo este influye en la construcción de su $\mathrm{ETM}_{\mathrm{G}}$.

En la concepción de Balacheff (1987) relativa al proceso de prueba, el autor distingue entre las pragmáticas y las intelectuales, y una tipología propia para cada una de estas 
pruebas, las cuales se diferencian por el estatus de los conocimientos en cuestión y la naturaleza de la justificación subyacente.

En relación a la formulación de los paradigmas geométricos, Houdement y Kuzniak $(1999,2006)$ identifican tres tipos paradigmas que coexisten en la enseñanza de la geometría, los cuales organizan la interacción entre intuición, experiencia y razonamiento. Desde esta perspectiva podemos observar cómo un sujeto reflexiona de acuerdo a las creencias, técnicas y el conocimiento de distintos modelos geométricos cuando desarrolla una tarea específica, es decir, que el $\mathrm{ETM}_{\mathrm{G}}$ puede variar dependiendo del paradigma geométrico dominante.

En el paradigma Geometría Natural (GI), la geometría se desarrolla sobre objetos reales u objetos materiales, con ayuda de la percepción y manipulación de instrumentos, y por tanto, la importancia en la aproximación y la medida; la construcción y la percepción están en el centro de esta geometría de tipo experimental.

En el paradigma Geometría Axiomática Natural (GII), la geometría se construye sobre un modelo próximo a la realidad e intuición espacial. El razonamiento de validación se funda sobre las leyes hipotéticas deductivas del sistema axiomático en juego, el que puede ser incompleto; los objetos geométricos son descritos por su definición o una propiedad.

La Geometría Axiomática Formal (GIII) se caracteriza por la separación entre la axiomática y la realidad. Los axiomas no están basados en lo sensible, el razonamiento de validación está basado en la coherencia lógica y formal por el sistema de axiomas del modelo subyacente; los objetos provienen de una axiomática con toda la rigurosidad y formalismo del modelo geométrico elegido.

Cabe señalar que la definición de estos paradigmas, particularmente GI y GII, conciernen al enfoque geométrico sintético. En el caso del enfoque analítico (cartesiano), se ha considerado una ampliación para caracterizar los paradigmas y favorecer su empleo en los análisis, lo que será presentado en las secciones 4.2.2 y 5.2 de los análisis a priori y análisis de resultados, respectivamente.

Finalmente, es posible analizar diferentes rutas de trabajo, lo que en el ETM se ha denominado una circulación entre las componentes de los planos cognitivo y epistemológico (MONTOYA-DELGADILLO; MENA-LORCA; MENA-LORCA，2014; HENRÍQUEZ; MONTOYA, 2015). En otras palabras, esta noción es relativa a la articulación entre los planos del ETM mediante las génesis, especificando las componentes puestas en juego. En este estudio se muestran resultados del diseño y experimentación de situaciones de referencia y, particularmente, el ETM personal de un profesor de matemática, mediante el estudio de la circulación entre las componentes de los planos en su ETM $_{\mathrm{G}}$. Las situaciones de referencia 
que han sido implementadas tienen la intención de integrar las tres génesis, lo cual implica coordinar aspectos semióticos con procesos de construcción, y razonamientos argumentativos y deductivos. En términos de paradigmas geométricos el objetivo ha sido favorecer GII, o bien, el tránsito entre GI y GII -aquí es visto como una etapa intermedia entre GI y GII que sedenota GI/GII.

En el apartado siguiente, se muestra partedel estudio al $\mathrm{ETM}_{\mathrm{G}}$ de referencia e idóneo del profesor de secundaria (HENRÍQUEZ, 2014). Estos análisis han proporcionado información para el diseño de las situaciones y evidencia empírica sobre el tema.

\section{Elementos de contextualización: ETM $_{G}$ del profesor}

En esta sección se analizan de manera general elementos que caracterizan el trabajo geométrico del profesor de secundaria y se identifica el paradigma geométrico privilegiado en el caso del ETM idóneo de un docente.

En una aproximación al $\mathrm{ETM}_{\mathrm{G}}$ de referencia del profesor se analiza el currículo escolar chileno, específicamente, sobre contenidos del eje geometría y cómo estos relacionan los enfoques geométricos euclidianos con y sin coordenadas cartesianas. En relación al ETM $\mathrm{G}_{\mathrm{G}}$ idóneo del profesor se presenta una parte de un estudio sobre la práctica en el aula de profesores cuando las temáticas se desarrollaron en uno u otro enfoque (HENRÍQUEZ; MONTOYA, 2015). En este caso, el interés fue estudiar las transposiciones realizadas por profesores de secundaria para la enseñanza de la geometría, las tareas que propone durante la clase y el ETM $\mathrm{G}_{\mathrm{G}}$ desarrollado en torno a dichas tareas.

\subsection{El caso del currículo escolar}

El currículo escolar del Ministerio de Educación (MINEDUC, 2009, p. 146) en el eje Geometría señala que “(...) amplía la base epistemológica de la geometría, mediante las trasformaciones rígidas en el plano, los vectores y la geometría cartesiana”. Lo anterior se expresa en los contenidos hasta $8^{\circ}$ Básico (13 años) sin el uso de coordenadas, predominando temas referidos a elementos básicos de la geometría euclidiana, transformaciones de figuras planas utilizando herramientas, entre otros.

En $1^{\circ}$ Medio (14 años), se estudia geometría en el plano cartesiano para representar puntos, figuras, vectores e isometrías. En $2^{\circ}$ Medio (15 años), los contenidos no consideran las coordenadas, como en semejanza de figuras planas. Cabe destacar que hasta este nivel, el 
tema de los enfoques geométricos y su complementariedad se presenta invisible para el docente.

En $3^{\circ}$ Medio (16 años) aparece el enfoque analítico en uno de los Objetivos Fundamentales "Comprender la geometría cartesiana como un modelo para el tratamiento algebraico de los elementos y relaciones entre figuras geométricas” (MINEDUC, 2009, p. 188). Sin embargo, no hay información que oriente el trabajo didáctico del docente en relación al tránsito y complementariedad entre los enfoques, ni cómo enseñarlos, es decir, la problemática persiste invisible para el profesor y la transposición que debe (o puede) realizar.

Finalmente, en $4^{\circ}$ Medio (17 años), se estudian las primeras nociones de geometría analítica del espacio -el pasaje del plano al espacio no es abordado aquí.

\subsection{El profesor y su práctica en el aula}

En una aproximación al ETM idóneo del profesor de secundaria, el trabajo de Henríquez y Montoya (2015) muestra los análisis de la práctica en aula de dos profesores en temas que conciernen a los enfoques geométricos sintético y analítico. El propósito del estudio no ha sido generalizar respecto al ETMG idóneo; sin embargo, los resultados muestran un diagnóstico relativo al trabajo geométrico del profesor en el nivel secundario. Este estudio, presentado en una publicación de la revista Enseñanza de las Ciencias, contribuyó significativamente al diseño de la situación de referencia propuesta en la presente investigación, como una alternativa que favorece la complementariedad entre los enfoques geométricos. Asimismo, dicho trabajo muestra la utilidad del ETM como constructo teórico para el análisis de la actividad matemática, en términos de las circulaciones favorecidas, especialmente, como una herramienta para el estudio en la formación de profesores.

Los análisis anteriores han proporcionado información y evidencia empírica respecto a la discontinuidad entre los enfoques sintético y analítico, reportando, entre otros aspectos, un privilegio por el trabajo en el paradigma GI. Los profesores relegan del trabajo la génesis discursiva de la prueba, consideran débilmente razonamientos argumentativos sustentados en elementos de la componente referencial, las justificaciones y validaciones de los procedimientos desarrollados no se fundamentan en propiedades y definiciones que favorecen los razonamientos de validación. Las génesis semiótica e instrumental fueron las más activadas por los profesores, privilegiando el plano vertical [Sem-Ins] (KUZNIAK; RICHARD, 2014). En uno de los casos, el trabajo en el dominio geométrico sintético cambia 
a otro dominio sin retorno al dominio original. En otro caso, en el trabajo según el enfoque con coordenadas, las génesis activadas no son articuladas.

En general, los análisis muestran que, cuando se activa la génesis instrumental, está centrada en el uso de un tipo de artefacto simbólico para dar solución al problema. Para coordinar el enfoque sintético con el analítico, el uso de signos y la semiótica intencionada son aspectos necesarios que favorecen el tránsito del enfoque sintético al analítico. En efecto, la vigilancia didáctica es fundamental, pues si bien el trabajo podría contribuir, también se podría caer en los cálculos ciegos al resolver ecuaciones, perdiendo el sentido del trabajo geométrico en sí y provocando un cambio de dominio (de geométrico a aritmético/algebraico), sin retorno al dominio fuente, como se reporta en el trabajo de Montoya-Delgadillo y Vivier (2014). En síntesis, se puede concluir que bajo el escenario analizado resulta difícil pensar en el tránsito y la complementariedad entre los enfoques geométricos.

En la siguiente sección se presentan elementos metodológicos empleados en relación al diseño y aplicación de las situaciones.

\section{Aspectos metodológicos}

\subsection{Características generales y selección de casos}

El propósito del estudio consiste en favorecer el tránsito y la complementariedad entre los enfoques sintético y analítico. Para esto fueron diseñadas y aplicadas situaciones de referencia para profesores de nivel secundario. En términos del ETM, la intención es integrar las tres génesis, en particular, favorecer el proceso de visualización no-icónica en coordinación con razonamientos discursivos, privilegiando el trabajo en GII, o bien, el tránsito entre GI y GII.

Para este estudio se escogieron métodos cualitativos de investigación, se realizaron grabaciones y sus respectivas transcripciones de los diálogos entre profesores durante la implementación de las situaciones, observaciones de sesiones no participante, el estudio al hábitat del problema en la Enseñanza Media (Secundaria) y la revisión de antecedentes sobre el tema.

En relación al diseño de las tareas y su vínculo con el ETM, Kuzniak (2011, p. 13) afirma que “(...) los problemas no son parte del espacio de trabajo pero son su razón de ser y su activador". Por esta razón, para analizar el ETM $_{\mathrm{G}}$ personal de profesores en situaciones que provocan el tránsito entre los enfoques geométricos, resultó fundamental el diseño de las 
situaciones de referencia que favorecen dicho propósito. Sobre las temáticas involucradas, fueron considerados elementos básicos de la Geometría Euclidiana en el plano tales como puntos, rectas, trazos; figuras planas tales como polígonos y circunferencias y, además, propiedades y definiciones sobre dichos elementos primarios. En una etapa de preexperimentación fueron diseñadas y aplicadas distintas situaciones a 3 estudiantes en último año de pedagogía en matemática de una universidad chilena (noviembre de 2013). Lo anterior permitió determinar qué situaciones aportaban mayor información para la investigación, posteriormente fueron refinadas las que se usaron en la fase de experimentación. Algunos aspectos relevantes fueron considerar el grado de dificultad, las temáticas involucradas, el tiempo que tardaban en resolver y el uso de artefactos -no serán presentados estos resultados.

La fase de experimentación se desarrolló entre diciembre de 2013 y enero de 2014. Las sesiones fueron desarrolladas en la universidad de los participantes, o bien, en su lugar de trabajo. La duración fue de 120 minutos -aproximadamente 60 minutos por situación-, y no se les indicó previamente qué tópicos estaban involucrados. Los participantes fueron clasificados según su experiencia en los siguientes tres grupos:

- Futuro profesor (FP): estudiantes de formación inicial en último año de pedagogía en matemática, que poseen experiencia como profesor de secundaria.

- Profesor debutante (PD): profesor de matemática de secundaria con hasta 3 años de ejercicio profesional.

- Profesor experimentado (PE): profesor de matemática de secundaria con más de 7 años de ejercicio profesional.

La formación inicial de los profesores se desarrolló en universidades chilenas públicas y una institución privada. La información general de los profesores participantes se presenta en la siguiente tabla (1).

Tabla 1 - Población de profesores participantes.

\begin{tabular}{|l|l|l|l|}
\hline Profesor & $\begin{array}{l}\text { Universidad } \\
\text { privada }\end{array}$ & $\begin{array}{l}\text { Universidad } \\
\text { pública }\end{array}$ & Total \\
\hline FP & 6 & 9 & 15 \\
\hline PD & 0 & 2 & 2 \\
\hline PE & 0 & 2 & 2 \\
\hline
\end{tabular}

Del total (19) de la población participante, 11 utilizaron regla y compás, y 8 el software Geogebra. La elección del tipo de artefacto fue de exclusiva voluntad de los profesores participantes. En este trabajo se abordan aquellos que consideraron el artefacto tecnológico, pues una de las preguntas tiene mayor riqueza utilizando el potencial dinámico 
del software. Esto implica favorecer el plano [Sem-Dis] en el $\mathrm{ETM}_{\mathrm{G}}$ mediante la instrumentalización de tipo dinámica por el uso del programa geométrico.

Los análisis de la situación de referencia aquí presentada se clasificaron según tipologías de respuestas en el trabajo sintético y analítico del ETM personal de todos los participantes. Reportamos aquí un caso (FP1) de los futuros profesores entre los 19 participantes, que consideramos relevante por lo manifestado en su ETM $_{\mathrm{G}}$ personal: FP1 es excepcional por ser el único caso según su tipología con el uso de artefacto, además, su trabajo obedeció a lo esperado en términos del paradigma privilegiado.

\subsection{Presentación de las situaciones de referencia}

Como una alternativa para la enseñanza o para su análisis en la formación de profesores se proponen situaciones que favorecen el tránsito y la complementariedad entre los enfoques sintético y cartesiano; se han diseñado dos situaciones de referencia que coordinan las génesis del $\mathrm{ETM}_{\mathrm{G}}$, en particular, favorecer el proceso de visualización no-icónica del modo constructeur e inventeur-bricoleur ${ }^{1}$ (DUVAL, 2005) en coordinación con razonamientos discursivos y uso de artefactos, privilegiando el trabajo en el paradigma geométrico GII (o bien, el paso de GI a GII).

Cada secuencia fue formulada como un protocolo de acción, en el cual los participantes debían desarrollar un plan de trabajo detallado. En este escrito, presentamos una de las situaciones en la modalidad con uso de software Geogebra, la cual consta de tres partes: primero, en versión sintética, segundo, una versión cartesiana y, tercera, preguntas abiertas. A continuación, se presenta en este artículo una situación de lugar geométrico.

En relación a las temáticas involucradas, se han considerado elementos básicos de la Geometría Euclidiana en el plano tales como puntos, rectas, trazos, figuras planas, propiedades y definiciones de dichos elementos primarios. El criterio utilizado para la selección obedece a que pueden ser utilizados y los enunciados adaptados en ambos enfoques y, además, se trata de nociones que están presentes en el currículo de educación primaria y secundaria.

\subsubsection{Versión sintética}

\footnotetext{
${ }^{1}$ Se dejaron las palabras en el idioma original pues la traducción nos parece no ser la más pertinente al español.
} 
La primera parte de la situación corresponde a la tarea formulada en versión sintética presentada en la siguiente tabla (2).

Tabla 2 - Tarea en versión sintética.

Dados dos puntos A y $B$ en el plano, construir el lugar geométrico de los puntos del plano que
equidistan de A y B.
Para ello use un software dinámico y solo utilizando las herramientas "Intersección de dos objetos"
, Recta que pasa por dos puntos" "Circunferencia dado su centro y uno de sus puntos"
1. Describa paso a paso la construcción realizada.
2. ¿Cómo justifica que la construcción realizada es la correcta? ¿Qué definiciones y/o propiedades
aparecen involucradas?
3. Si se movieran los puntos A y B ¿la solución sigue teniendo validez? Justifique su respuesta.

En esta versión sintética de la tarea, en el trabajo geométrico mediado por el uso de Geogebra, se espera favorecer el potencial dinámico que entrega dicho artefacto, específicamente, en la pregunta 3 para validar los procedimientos de la construcción realizada. Por otro lado, al restringir las herramientas del software, en particular las mediciones, el trabajo en el paradigma GI queda limitado.

En las posibles soluciones se han considerado la construcción de triángulos equiláteros (del Libro I: Proposición I de los Elementos de Euclides) dados los puntos A y B en el plano. Luego, dibujar la circunferencia de centro A y radio AB utilizando la herramienta $\odot$-o bien, es posible considerar radios superior a la mitad de la longitud de $\mathrm{AB}-$. El mismo procedimiento para dibujar la circunferencia con centro en B y uno de sus puntos es $\mathrm{A}$, como se muestra en Figura 1: imagen 1. La intersección de ambas circunferencias determina los puntos C y D, utilizando la herramienta (Figura 1: imagen 2), y dichos puntos equidistan de A y B. Luego, la recta que pasa por los puntos C y D es el lugar geométrico solicitado, trazada con la herramienta (Figura 1: imagen 3).

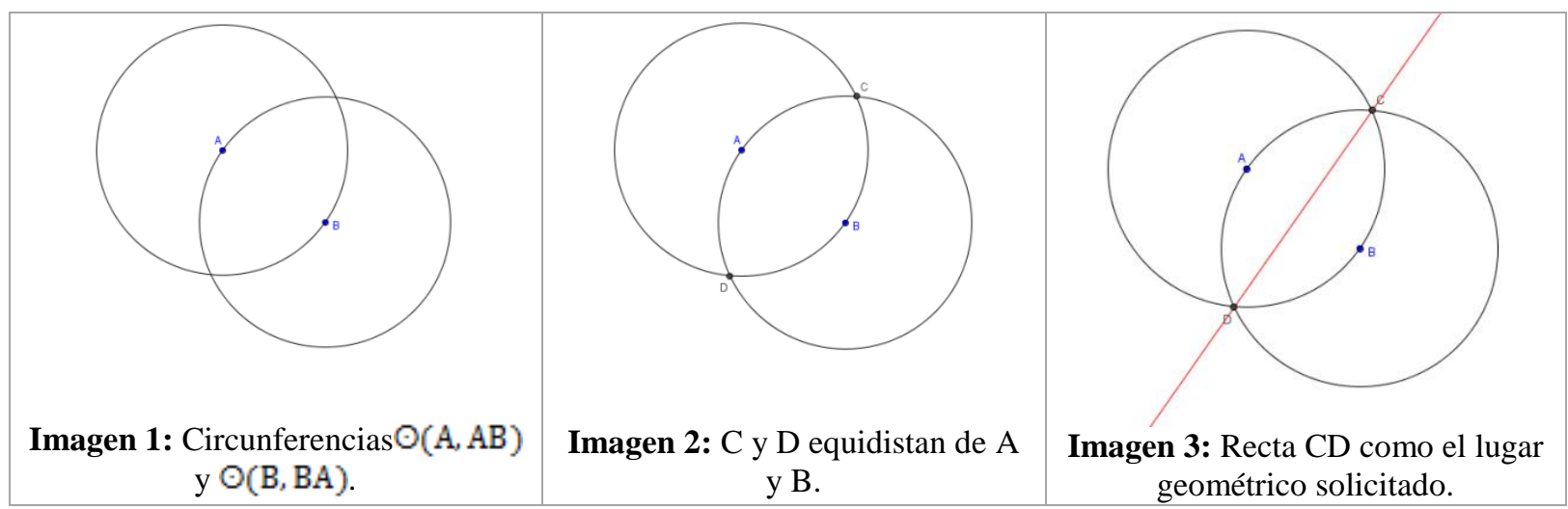

Figura 1 - Secuencia del trabajo geométrico con GeoGebra. 
Según la construcción desarrollada, se tiene que $\mathrm{AC}=\mathrm{BC}$, entonces $\mathrm{C}$ pertenece a la mediatriz del segmento $\mathrm{AB}$. Además, $\mathrm{AD}=\mathrm{BD}$, entonces $\mathrm{D}$ pertenece a la mediatriz del segmento AB. Los puntos $\mathrm{C}$ y D son dos puntos distintos de la mediatriz. Por tanto, la recta que pasa por $\mathrm{C}$ y $\mathrm{D}$ es la mediatriz del trazo $\mathrm{AB}$. Luego, todo punto que pertenece a la mediatriz (recta CD), está a igual distancia de A y B, lo cual soluciona el problema.

Desde un punto de vista teórico, en esta tarea se privilegia el modo de visualizar constructeur. Inicialmente, es activado el proceso de construcción, a través del uso de herramientas que proporciona el software y las operaciones realizadas en cierto orden. Estos procedimientos de construcción se deben comunicar, activando así la génesis discursiva. La pregunta 2 coordina la génesis instrumental con la génesis discursiva, pues es preciso justificar la técnica de construcción. En la justificación de la construcción aparecen definiciones (como circunferencia, mediatriz) y propiedades (como de mediatriz, de paralelogramo). En términos de prueba, el trabajo tiene características de pragmática e intelectual, específicamente, del tipo ejemplo genérico (BALACHEFF, 1987). La validación de la construcción es activada en la pregunta 3, aprovechando el potencial dinámico del software. El trabajo favorece la génesis discursiva de la prueba sobre una configuración previamente construida.

En términos del paradigma geométrico privilegiado, se trata de una forma intermedia entre GI y GII (GI/GII), pues si bien el trabajo favorece el uso de artefactos, estos son utilizados para construir una configuración geométrica y, luego, en la elaboración de la justificación y la validación de la construcción se deben reconocer y enunciar propiedades.

\subsubsection{Versión analítica}

En esta versión se presenta la tarea formulada en versión analítica (cartesiana), la cual se muestra en la siguiente tabla (3).

Tabla 3 - Tarea en versión analítica.

Dados dos puntos $A(0,0)$ y $B(2,2)$ en el plano, determinar algebraicamente el lugar geométrico de los puntos que equidistan de $A$ y $B$.

1. ¿Cómo justifica que la expresión algebraica es la correcta? ¿Qué definiciones y/o propiedades aparecen involucradas?

En este caso, se trata de una versión analítica particular de la versión sintética anterior. La decisión de usar software (o no) depende de los participantes. La elección de puntos, que en este caso fueron $\mathrm{A}(0,0)$ y $\mathrm{B}(2,2)$, considera dos aspectos relevantes: inicialmente permitir ver la solución si es que los participantes grafican, favoreciendo la continuidad entre el trabajo 
en versión sintética anterior y la presente versión cartesiana. Además, facilitar los tratamientos algebraicos, pues en este caso no es la intención del problema que la dificultad esté en dichos tratamientos, sino en la recta solución, su justificación y la activación de la componente referencial favoreciendo el uso de propiedades que en la versión sintética no fue preciso emplear.

En las posibles estrategias se ha considerado graficar (inicialmente) los puntos dados A y B (Figura 2: imagen 1) en el sistema de coordenadas cartesianas rectangular. De forma intuitiva, se puede esbozar el lugar geométrico pedido; sin embargo, esta no es la solución esperada. En la solución esperada, si $\mathrm{P}(x, y)$ es un punto del lugar geométrico, se tiene: $d(\mathrm{P}, \mathrm{A})=d(\mathrm{P}, \mathrm{B})$; donde $d$ representa la distancia entre dos puntos. Luego, como $\mathrm{A}(0,0) \mathrm{y}$ $\mathrm{B}(2,2)$, se tiene la distancia entre dos puntos:

$$
\begin{aligned}
& d[(x, y),(0,0)]=\sqrt{(x-0)^{2}+(y-0)^{2}} \\
& d[(x, y),(2,2)]=\sqrt{(x-2)^{2}+(y-2)^{2}}
\end{aligned}
$$

$\mathrm{Al}$ igualar se tiene que las cantidades subradicales son positivas, se eleva al cuadrado y se obtiene: $x^{2}+y^{2}=x^{2}-4 x+4+y^{2}-4 y+4$. Al resolver resulta la recta: $x+y-2=0$. Por lo tanto, los puntos $\mathrm{P}(x, y)$ son de la forma $\mathrm{P}(x,-x+2)$, donde $x$ es un número real.

Al graficar la recta obtenida es posible verificar que se trata de la mediatriz del segmento $\mathrm{AB}$ (Figura 2: imagen 2), y al utilizar el software, la vista algebraica permite comprobar que se trata de la misma recta obtenida (Figura 2: imagen 3).

Para responder la pregunta 1 , es posible saber del trabajo sintético anterior, que el lugar geométrico es la mediatriz del segmento $\mathrm{AB}$. Luego, se puede justificar algebraicamente que la recta es la solución, es decir que pasa por el punto medio del segmento y que es perpendicular a él. También se puede determinar la ordenada (o abscisa) de un punto en la recta usando su representación algebraica y verificar que pertenecen a la recta por su representación gráfica.

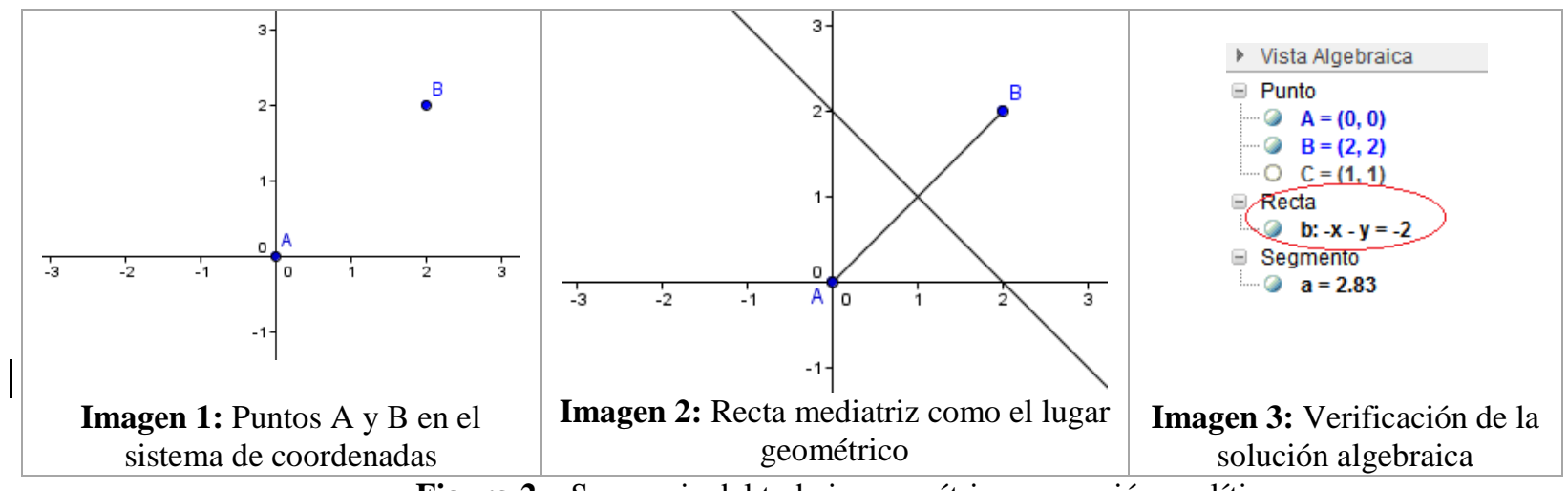

Figura 2 - Secuencia del trabajo geométrico en versión analítica. 
Desde la perspectiva teórica, inicialmente el trabajo implica graficar los puntos dados A y B, se trata de un caso particular del problema. De este modo, el trabajo activa la visualización con una representación gráfica, pero esto no da solución al problema, es necesario realizar tratamientos en el registro algebraico en coordinación con la visualización de la gráfica cartesiana. No obstante lo anterior, es posible que no se efectúe dicha coordinación, realizando solo el trabajo algebraico.

En los tratamientos algebraicos se debe utilizar un teorema como un artefacto simbólico, la fórmula de la distancia entre dos puntos, lo cual permite obtener la recta que es la solución del problema. En efecto, aquí la componente referencial es movilizada en la determinación de la recta solución y, además, en la justificación que precisa otras nociones matemáticas en su desarrollo (perpendicularidad de rectas, entre otros). La tipología de prueba en este caso tiene características del tipo intelectual, específicamente experimento mental (BALACHEFF, 1987).

En términos del paradigma geométrico privilegiado, al igual que el caso sintético, se trata de una fase intermedia entre GI y GII, pero en versión cartesiana. Esto se funda, por un lado, en la localización de puntos específicos para representar objetos geométricos en el sistema de coordenadas y, por otro, en las ecuaciones, los tratamientos algebraicos, la elaboración del razonamiento discursivo en la justificación y elementos del referencial que son empleados como artefactos simbólicos (en este caso es un teorema). Cabe señalar que, en esta tarea ambos trabajos geométricos se coordinan; sin embargo, el trabajo con ecuaciones podría prescindir de los gráficos.

En síntesis, la intención de la situación es coordinar los enfoques geométricos. Asimismo, acrecentar el referencial en relación a un mismo objeto matemático.

\subsubsection{Preguntas al profesor}

En la tercera parte, se presentan las siguientes preguntas, las cuales se muestran en la siguiente tabla (4).

Tabla 4 - Preguntas al profesor.

De la pregunta 1 (versión sintética) y la pregunta 2 (versión analítica), responda:
1 ¿QQué consecuencias o qué características se pueden extraer del lugar geométrico que ha determinado?,
¿cómo lo justifica en ambos casos?
2. En su rol de profesor, y considerando a sus (futuros) estudiantes de Enseñanza Media ¿le parece que
un procedimiento es más apropiado que el otro?, ¿por qué? Explicite su respuesta.


La última parte de esta secuencia, y a través de dos preguntas abiertas, sitúa al profesor desde la perspectiva de la enseñanza: su $\mathrm{ETM}_{\mathrm{G}}$ idóneo. En ambas preguntas, la intención es provocar la comparación entre ambos enfoques geométricos y que el docente se refiera a la posibilidad de realizar ambos trabajos en continuidad y complementariedad en la enseñanza.

\section{Análisis de resultados}

A continuación, se muestran los análisis relativos al $\mathrm{ETM}_{\mathrm{G}}$ personal desarrollados por FP1, según ambas versiones de la tarea. Presentamos la circulación entre las componentes de los planos cognitivo y epistemológico, y la activación de las génesis de los $\mathrm{ETM}_{\mathrm{G}}$ sintético y analítico. Como fue mencionado más arriba, FP1 es el único caso según su respuesta con el uso de artefacto, además, su trabajo resulta interesante de mostrar en términos del paradigma privilegiado.

\subsection{Versión sintética}

El trabajo de FP1 se caracteriza por privilegiar (inicialmente) la articulación entre las génesis figural e instrumental. El trabajo consiste en trazar una recta que pasa por A y B dados, luego dos circunferencias de centro A y B, respectivamente -sin aludir a sus radios. En seguida, FP1 usa la herramienta de intersección de dos objetos para marcar los puntos C y D (Figura 3: imagen 1). Luego, traza la recta que pasa por C y D, e indica:

FP1: Visualmente puedo presumir que la recta $\mathrm{b}$ es el posible lugar geométrico, por lo que debo demostrarlo a continuación.

Para dar respuesta a las preguntas 2 y 3, FP1 articula la génesis figural (en el proceso de visualización) y la génesis discursiva, apoyado en la construcción de un paralelogramo y las propiedades de sus diagonales (Figura 3: imagen 2). En este caso, aprovecha el potencial dinámico del software para deslizar puntos de la construcción. Finalmente, concluye que la recta $\mathrm{CD}$ es mediatriz de la recta $\mathrm{AB}$. 


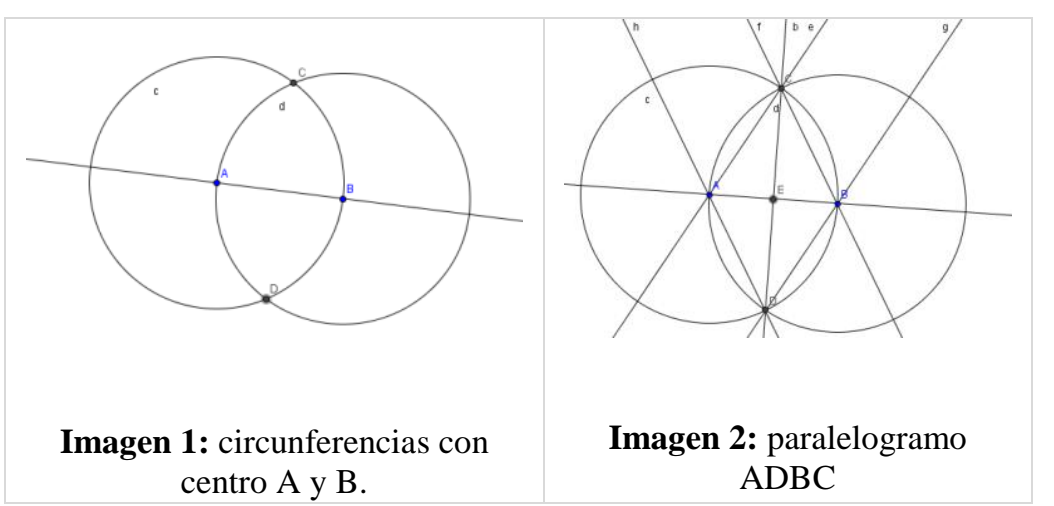

Figura 3 - Imágenes del trabajo sintético realizado por FP1.

Para justificar el procedimiento de construcción, FP1 se apoya en que ADBC es un paralelogramo y en propiedades obtenidas por experimentación, para justificar que la mediatriz del segmento $A B$ es perpendicular al trazo y lo biseca. Además, señala que la construcción siempre tiene validez pues depende del segmento $\mathrm{AB}$, lo cual varía según sea el radio de las circunferencias.

En el caso del trabajo sintético de FP1, se observa que privilegia el paradigma GII, puesto que la justificación de la construcción realizada se funda en propiedades de figuras y definiciones. En este sentido, el trabajo es realizado en concordancia con el propósito de la situación planteada, es decir, favorecer una forma de visualización no-icónica (constructeur) en coordinación con razonamientos discursivos para validar el trabajo, donde elementos específicos del referencial (congruencia de triángulos, teorema de congruencia, definición de mediatriz y propiedades del rombo) son los empleados y la visualización de la configuración es un apoyo en el trabajo discursivo. La utilización del programa (software) para la construcción nos permite afirmar que FP1 ha instrumentalizado el artefacto, y con esto, la génesis instrumental se activa y articula con las génesis figural y discursiva, respectivamente.

\subsection{Versión analítica}

En este caso, FP1 inicialmente grafica (en una hoja) los puntos dados A, B y un punto C ubicado en el punto medio del segmento AB (Figura 4: imagen 1). La versión sintética ayuda a ver que el lugar geométrico pasa por C. Además, FP1 señala que se trata de una recta. El trabajo se desarrolla a partir de la visualización de los puntos, que luego coordina con un trabajo semiótico, y proponemos el uso de distancia entre dos puntos como un artefacto simbólico, en el desarrollo de tratamientos en el registro algebraico (Figura 4: imagen 2). Así, el trabajo que inicialmente es ver, favorece la génesis semiótica y ciertos elementos de la 
componente referencial, propiciando una conversión entre las representaciones en registro geométrico (o gráfico) y en registro algebraico.

Este caso, que involucra una versión particular (y un trabajo sintético anterior que ayudó a graficar), presenta elementos del paradigma GI por la localización de puntos y el énfasis en lo que se ve de la representación gráfica. Sin embargo, hay un tipo de trabajo semiótico en términos de tratamiento y conversión (en registro algebraico) desarrollado por FP1, que es justificado con un teorema (distancia entre dos puntos); por lo cual se postula que el trabajo presenta también características del paradigma GII. En este sentido, se considera un ETM en versión cartesiana que favorece el paso de GI a GII.

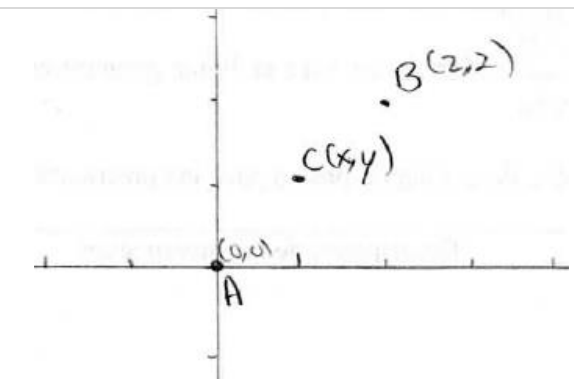

Imagen 1: puntos $\mathrm{A}, \mathrm{B}$ y $\mathrm{C}$ en el plano cartesiano.

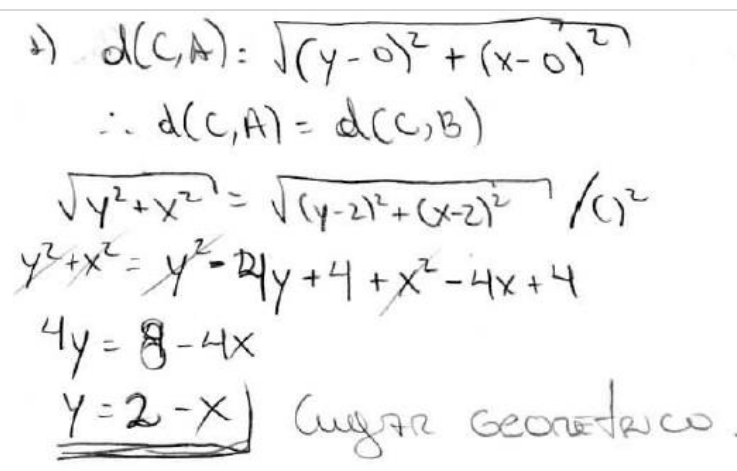

Imagen 2: trabajo algebraico en el enfoque analítico.

Figura 4 - Imágenes del trabajo cartesiano realizado por FP1.

\subsection{Preguntas al profesor}

En esta sección se muestran análisis de las respuestas proporcionadas por los profesores en términos generales, independiente de la tipología de respuesta, paradigma geométrico privilegiado y de la validez (o no) de los resultados obtenidos.

La mayoría de los profesores sostiene que una vez desarrollada la situación en ambas versiones, el trabajo se complementa y es factible proponer en la enseñanza ambos enfoques geométricos. Igualmente se evidencian algunas excepciones, como dos casos de FP (distintos a FP1), quienes manifiestan que les parece más adecuado el trabajo analítico, por ser más formal y claro.

La mayor cantidad de los participantes destacan el potencial del enfoque sintético por permitir ver y construir, y del enfoque analítico, la posibilidad de representar la recta algebraicamente. Sin embargo, algunos señalan no haber planteado esta problemática antes en un sentido didáctico, y por ende, se deduce que esto no fue cuestionado en su formación inicial. 
Otro aspecto relevante tiene relación con la posibilidad de proponer situaciones de este tipo en la enseñanza; la mayoría señala que se deberían abordar en distintos niveles. Por ejemplo, un FP destaca que la versión analítica debería ser enseñada en un nivel superior escolar al sintético visto en educación básica, pues se requiere de conocimientos algebraicos que en ese nivel los estudiantes no poseen. Solamente un profesor destaca las ventajas en términos de visualización usando el software, argumentando que al utilizar GeoGebra y mover la figura, realmente se puede ver el lugar geométrico.

\section{Conclusión}

Estudios anteriores desarrollados en el marco del ETM respecto a la práctica en el aula del profesor de secundaria en temas de geometría, han evidenciado el predominio del trabajo aritmético/algebraico, la visualización icónica y poco énfasis en los razonamientos discursivos. A lo anterior, se suma la falta de orientaciones didácticas para el profesor que potencien un $\mathrm{ETM}_{\mathrm{G}}$ personal en los estudiantes. En este trabajo aportamos con una propuesta que favorece la complementariedad entre los enfoques sintético y cartesiano, considerando la articulación entre las génesis del ETM. Asimismo, se presentan los análisis al diseño, se proporcionan datos de la etapa de experimentación y de los análisis de un caso, evidenciando el potencial del ETM como herramienta de análisis al trabajo matemático.

En la situación de referencia presentada se desarrolla el proceso de construcción, la cual luego demanda su justificación y validación. En la versión sintética resaltamos la relevancia de la variable didáctica al restringir las herramientas del software, lo cual obligó a realizar una construcción geométrica y la visualización dinámica en la validación de la construcción.

Cabe destacar, que en los resultados se han identificado y distinguido dos fenómenos relevantes: bloqueos y dificultades en el ETM. El bloqueo alude a la presencia de un error en el trabajo o en alguna etapa del trabajo, que no permite dar una respuesta esperada, evidenciando falta de coordinación entre las génesis o en las componentes del ETM. Una dificultad se refiere a un problema en la articulación entre génesis o componentes del ETM, lo cual impide que se aproveche el potencial del trabajo en torno a las génesis involucradas.

En los resultados, según el enfoque sintético se observan 6 tipologías diferentes de respuesta, relativas a formas distintas de construcción, en las cuales se manifiestan dificultades ligadas a la génesis discursiva. Del total de participantes, dos casos (FP) de los 19, evidenciaron bloqueos en el ETM, en los cuales la validación de resultados apareció 
desarticulada de la construcción con un débil referencial, manifestado en el escaso rigor en el discurso y en el proceso de visualización. En el resto de los casos (17 de 19), aún cuando existieron diferencias según las tipologías de respuestas, las justificaciones y validaciones de procedimientos de construcción evidenciaron diferencias en la formalidad, identificando tránsitos entre paradigmas (GI/GII) y la presencia de GII. Específicamente, en las pruebas utilizadas, se observó una tipología intermediaria entre pragmático e intelectual, con mayor o menor énfasis de uno u otro.

La versión cartesiana presenta menos bloqueos y dificultades que la versión sintética, lo cual podría explicarse por el hecho que ya conocen su solución (de la versión sintética). Otra explicación a este hecho puede estar relacionada a que el ETM personal de los profesores se ha visto influenciado por el ETM idóneo y por el ETM de referencia. Particularmente, en las instituciones escolares chilenas hemos evidenciado una algebrización del trabajo geométrico.

Un aporte al ETM en este trabajo fue identificar y definir paradigmas geométricos en el enfoque cartesiano, propiciados por la situación de referencia en versión analítica (cartesiana).

En la tercera parte preguntas al profesor, cuando se pregunta por la posibilidad de llevar este tipo de trabajo a la enseñanza, la mayoría señala que los trabajos se complementan. Algunos se refieren al nivel de los estudiantes términos curriculares y su factibilidad según la organización oficial de los saberes escolares; sin embargo, bajo este criterio ya hemos constatado su discontinuidad. En dos casos que no fueron presentados en este trabajo (PE), queda de manifiesto que en su ETM idóneo no consideran la complementariedad entre enfoques. Además, declaran que es factible realizar el trabajo para cursos de educación secundaria electivo -los estudiantes optan por mas horas y especialización en matemática-, retomando contenidos de niveles anteriores para comparar los enfoques.

En las consideraciones para el ETM idóneo destacamos: el potencial dinámico del software en el trabajo de validación de resultados y en la formulación de conjeturas, en lo cual se podría aprovechar y considerar en la planificación de la enseñanza, en la práctica en el aula del profesor, en las acciones y formulaciones de cursos de formación de profesores. Esto pues evidenciamos que, aquellos profesores que trabajaron en la modalidad con uso de GeoGebra, no aprovechan los deslizamientos que son posibles y el potencial dinámico que en este entorno tecnológico se puede propiciar, aún cuando esto fue demandado en una de las preguntas. 
De acuerdo a los resultados vertidos por FP1, la situación de referencia logra que coordine ambos enfoques; el trabajo sintético ayudó en el desarrollo del trabajo cartesiano. El restringir las herramientas del software fue otro aspecto a destacar en la propuesta como variable didáctica, pues obligó a realizar una construcción y favorecer la visualización que a priori se pretendía. En la tercera parte de la situación, cuando se pregunta por la posibilidad de llevar este tipo de trabajo a la enseñanza secundaria, FP1 se refiere al nivel de los estudiantes términos del currículo, y señala que estos enfoques son enseñados en forma discontinua.

Por otro lado, se pudo evidenciar que FP1 no distingue diferentes tipos de prueba, cuestión esencial para el desarrollo de los razonamientos discursivos y la coordinación con el proceso de visualización en geometría. Este asunto, ha quedado plasmado en el trabajo de Montoya-Delgadillo (2014) y no ha sido del todo resuelto en la formación de profesores en Chile. Un trabajo pendiente es trabajar en propuestas en que la génesis discursiva sea privilegiada articulando con las otras génesis del ETM.

En general, la situación de referencia presentada permitió cumplir con el objetivo en términos de tránsito y complementariedad entre los enfoques. Además, los profesores participantes enriquecieron su $\mathrm{ETM}_{\mathrm{G}}$ personal, lo cual fue evidenciado en la tercera parte de la situación. Nosotros sugerimos que la génesis discursiva en coordinación con el proceso de visualización (actualmente conocido como plano [Sem-Dis]) debe ser intencionado en términos de transposición, de modo que permita tomar acciones concretas para generar circulaciones específicas en el ETM.

Por último, consideramos que es posible extender la investigación, abarcar otras temáticas y otro tipo de tareas. Por ejemplo, mirar la geometría desde el punto de vista del grupo de transformaciones geométricas, en alusión al punto de vista de Klein es una oportunidad para continuar en esta línea de investigación. Asimismo, considerar cómo contribuir e influir en el $\mathrm{ETM}_{\mathrm{G}}$ idóneo de un profesor de liceo para coordinar los enfoques y la posibilidad de llevarlo a su práctica en el aula es un desafío latente para la formación inicial y continua de profesores.

\section{Agradecimientos}

Este trabajo fue financiado parcialmente por los proyectos FONDECYT N¹110988, beca doctoral y postdoctoral otorgada por la Pontificia Universidad Católica de Valparaíso, Chile. 


\section{Referencias}

ARTIGUE, M. Learning mathematics in a CAS environment: the genesis of a reflection about instrumentation and dialectics between technical and conceptual work. International Journal of computer for Mathematical Learning, Netherlands, v. 7, n. 3, p. 245-274, oct. 2002.

BALACHEFF, N. Processus de preuve et situations de validation. Educational studies in mathematics, Dordrecht, v. 18, n. 2, p. 147-176, may. 1987.

BOURBAKI, N. (1969). Formas cuadráticas. Geometría elemental. In: HERNÁNDEZ, J.(Ed.). Elementos de historia de las matemáticas. Madrid: Alianza Editorial, 1969, p. 173-19.

CHILE. Ministerio de Educación-MINEDUC. Objetivos fundamentales y contenidos mínimos obligatorios para la formación general: matemática. In: Curriculum. Objetivos fundamentales y contenidos mínimos obligatorios de la educación básica y media: Actualización 2009. Santiago: MINEDUC, 2009, p. 145-194.

DUVAL, R. Sémiosis et pensée humaine : registres sémiotiques et apprentissages intellectuels. Berne: Peter Lang, 1995.

DUVAL, R. Representation, vision and visualization: cognitive functions in mathematical thinking. basic issues for learning. In: PSYCHOLOGY OF MATHEMATICS EDUCATION CONFERENCE, $\mathrm{n}$. 21st, v.1, 1999, México. Proceedings of the twenty-first annual meeting of the north american chapter of the international group for the psychology of mathematics education. Mexico: Editora, 1999, p. 3-26.

DUVAL, R. Les conditions cognitives de l'apprentissage de la géométrie: développement de la visualisation, diférenciation des raisonnements et coordination de leurs fonctionnements. Annales de Didactique et de Sciences Cognitives, Strasbourg, v. 10, p. 5-53. 2005.

GASCÓN, J. Geometría sintética en la ESO y analítica en el bachillerato. ¿Dos mundos completamente separados? Suma, Badalona, v. 39, n. 1, p. 13-25, feb. 2002.

GÓMEZ-CHACÓN, I.; KUZNIAK, A. Les espaces de travail géométrique de futurs professeurs en contexte de connaissances technologiques et professionnelles. Annales de Didactique et de Sciences Cognitives, Strasbourg, v. 16, p. 187-216. 2011.

HENRÍQUEZ, C. El trabajo geométrico de profesores en el tránsito de la geometría sintética a la analítica en el nivel secundario. 2014. 251. Tesis (Doctorado en Didáctica de la Matemática). Pontificia Universidad Católica de Valparaíso, Valparaíso, 2014.

HENRÍQUEZ, C.; MONTOYA, E. Espacios de trabajo geométrico sintético y analítico de profesores y su práctica en el aula. Enseñanza de las Ciencias, Barcelona, v. 33, n. 2, p. 51-70, jun. 2015.

HOUDEMENT, C.; KUZNIAK, A. Un exemple de cadre conceptuel pour de l'enseignement de la géométrie en formation des maîtres. Educational Studies in Mathematics, Dordrecht, v. 40, n. 3, p. 283-312, nov. 1999.

HOUDEMENT, C.; KUZNIAK, A. Paradigmes géométriques et enseignement de la géométrie. Annales de Didactique et de Sciences Cognitives, Strasbourg, v. 11, p. 175-193. 2006.

KLEIN, F. Matemática elemental: desde un punto de vista superior. Traducción de R. Fontanilla Madrid : Biblioteca Matemática, 1908. v.2. 
KUZNIAK, A. L'espace de travail mathématique et ses genèses. Annales de Didactique et de Sciences Cognitives, Strasbourg, v. 16, p. 9-24. 2011.

KUZNIAK, A.; RICHARD, P. Espaces de travail mathématique : puntos de vista y perspectivas. Revista Latinoamericana de Matemática Educativa, México, v. 17, n. 4-I, p. 5-15, dic. 2014.

MONTOYA-DELGADILLO, E. El proceso de prueba en el espacio de trabajo geométrico: profesores en formación inicial. Revista Enseñanza de las Ciencias, México, v. 32, n. 3, p. 227-247, dic. 2014.

MONTOYA-DELGADILLO, E.; MENA-LORCA, A.; MENA-LORCA, J. Circulaciones y génesis en el espacio de trabajo matemático. Revista Latinoamericana de Investigación en Matemática Educativa, México, v. 17, n. 4-I, p. 181-197, dic. 2014.

MONTOYA-DELGADILLO, E.; VIVIER, L. Les changements de domaine de travail dans le cadre des Espaces de Travail Mathématique. Annales de Didactique et de Sciences Cognitives, Strasbourg, v. 19, p. 73-101, 2014.

PEIRCE, C. Ecrits sur le signe. Paris: Seuil, 1978.

RABARDEL, P. Les hommes et les technologies: une approche cognitive des instruments contemporains. Paris: Armand Colin, 1995.

TROUCHE, L. Une approche instrumentale de l'apprentissage des mathématiques dans des environnements de calculatrice symbolique. In: GUIN, D.;TROUCHE, L. (Ed.). Calculatrices Symboliques. Transformer un outil du travail informatique: un problème didactique. Grenoble: La Pensée Sauvage, 2002, p.187-214.

Submetido em Julho de 2015. Aprovado em Setembro de 2015. 